ARTIGO ORIGINAL ORIGINAL ARTICLE

\title{
Custo por evento evitado da enzalutamida versus abiraterona associada à prednisona em homens com câncer de próstata resistente à castração metastático, sob a perspectiva do Sistema de Saúde Suplementar no Brasil
}

\author{
Cost of preventing an event (COPE) of enzalutamide \\ versus abiraterone plus prednisone in men with \\ metastatic castration-resistant prostate cancer under \\ the Brazilian private health care system perspective \\ Stephen Stefani ${ }^{1}$, Vanessa Teich², André Fay ${ }^{3}$, Marcia Abadi ${ }^{4}$, Scott Flanders 5 , \\ Cat Bui ${ }^{5}$, André Sasse ${ }^{6}$
}

\section{Palavras-chave:}

custos e análise de custo, neoplasias de próstata resistentes à castração, enzalutamida, abiraterona

\section{RESUMO}

Introdução: O câncer de próstata resistente a castração metastático (CPRCM) é definido como uma neoplasia proliferativa contínua. Com o avanço de novas tecnologias, novos tratamentos foram desenvolvidos: enzalutamida (ENZ) e acetato de abiraterona + prednisona (AA+P). Este estudo analisou o custo por evento evitado (COPE) da ENZ e AA+P no tratamento do CPRCM após quimioterapia, sob a perspectiva do Sistema de Saúde Suplementar, utilizando os desfechos de eficácia sobrevida global (SG) e sobrevida livre de progressão radiográfica (SLPr). Métodos: Uma análise de custo foi realizada baseada nos estudos pivotais de ENZ e AA+P após uso de docetaxel a fim de calcular o COPE. Dois parâmetros foram necessários: custo anual de tratamento (CAT) e número necessário a tratar (NNT). O NNT é o inverso da redução de risco absoluto (RRA). O COPE é o CAT multiplicado pelo NNT. Resultados: Os resultados de CAT destacam uma economia de R\$ 1.006,00 (ENZ: R\$ 73.799,00 vs AA+P: R\$74.804,00) por paciente com ENZ em comparação com AA+P. O NNT, em 12 meses, foi: $\mathrm{NNT}_{\mathrm{SG} / \mathrm{NZ}}=7,14 ; \mathrm{NNT}_{\mathrm{SPr} / \mathrm{NZ}}=5,00 ; \mathrm{NNT}_{\mathrm{SG} / \mathrm{AA}+\mathrm{P}}=8,33$ e NNT $\mathrm{NLr} / \mathrm{AA}+\mathrm{P}=8,33$. Assim, o COPE calculado foi: $\mathrm{COPE}_{\mathrm{SG} / \mathrm{ENZ}}=\mathrm{R} \$ 527.691,00 ; \mathrm{COPE}_{\mathrm{SLPr} / \mathrm{ENZ}}=\mathrm{R} \$ 369.384,00 ; \mathrm{COPE}_{\mathrm{SG} / \mathrm{AA}+\mathrm{P}}=\mathrm{R} \$ 623.368,00$ e COPE $A_{A+P}=R \$ 623.368,00$. Os resultados mostram uma diferença anual de $R \$ 95.677,00$ e $R \$ 253.984,00$ representando um aumento que varia de $18,13 \%$ a $68,76 \%$, dependendo do desfecho considerado. Conclusão: Os resultados apontaram que ENZ comparada à AA+P proporciona menor COPE (CO$\mathrm{PE}_{\mathrm{ENZ}}$ é menor do que COPE $\mathrm{AA+P}_{\mathrm{P}}$ em ambos os desfechos analisados: SG e SLPr).
Recebido em: 05/11/2015. Aprovado para publicação em: 23/11/2015

1. Instituto do Câncer Mãe de Deus, Rio Grande do Sul, Brasil / Chair do Consórcio Latino Americano da International Society for Pharmacoeconomics and Outcomes Research (ISPOR).

2. Sense Company, São Paulo, Brasil.

3. Instituto do Câncer Mãe de Deus, Rio Grande do Sul, Brasil / Faculdade de Medicina da Pontifícia Universidade Católica do Rio Grande do Sul (PUCRS), Rio Grande do Sul, Brasil.

4. Astellas Farma, São Paulo, Brasil.

5. Astellas Farma, Illinois, Estados Unidos

6. Centro de Evidências em Oncologia (CEVON) da Universidade Estadual de Campinas (UNICAMP), São Paulo, Brasil.

Instituição onde o trabalho foi executado: Sense Company, São Paulo, SP, Brasil

Informações sobre auxílios recebidos sob a forma de financiamento, equipamentos ou medicamentos: Este estudo foi financiado pela Astellas Farma Brasil. Não obstante, convém salientar que não houve qualquer interferência ou ingerência da referida empresa junto aos autores para a alteração de qualquer resultado obtido pelos autores.

Conflito de interesses: Enzalutamida é co-desenvolvido pela Astellas Pharma, Inc., Northbrook, IL, EUA e Medivation, Inc., San Francisco, CA, EUA. Esta pesquisa e assistência editorial no desenvolvimento deste manuscrito foram feitos pelos autores em colaboração com a Sense Company e foram financiados pela Astellas Brasil.

Autor correspondente: Vanessa Teich, Rua Ministro Jesuíno Cardoso, 454, 70 andar, Ed. The One - Vila Nova Conceição, São Paulo / SP-04544-051 -E-mail: vanessa.teich@wearesense.company 


\section{Keywords:}

costs and cost analysis, prostatic neoplasms, castration-resistant, enzalutamide, abiraterone

\begin{abstract}
Introduction: Metastatic castration-resistant prostate cancer (mCRPC) is defined as a continuous proliferative cancer. With the advancement of new technologies, new treatments were developed: enzalutamide (ENZ) and abiraterone acetate plus prednisone (AA+P). This study examined the cost of preventing an event (COPE) with ENZ and AA+P in the treatment of $\mathrm{mCRPC}$ after chemotherapy, from the perspective of the Supplementary Health System, considering the clinical outcomes overall survival (OS) and radiographic progression-free survival (rPFS). Methods: A cost analysis was performed on post-docetaxel pivotal studies for ENZ and AA+P, in order to calculate the COPE. For that two parameters were required: annual treatment cost (ATC) and number needed to treat (NNT). NNT is the inverse of the Absolute Risk Reduction (ARR). COPE is the ATC multiplied by the NNT. Results: The ATC results indicated a BRL1,006 (ENZ: BRL73,799 vs AA+P: BRL74,804) saving per patient treated with ENZ compared to AA+P. NNT, in 12 months, was: NNT OS/ENZ $=7.14$; NNT rPFS/ENZ $=5.00$; $N N T_{\text {OS/AA+P }}=8.33$ and NNT rPFS/AA+P $=8.33$. Thus, the COPE calculated was: COPE ${ }_{\mathrm{OS} / \mathrm{ENZ}}=\mathrm{BRL} 527,691 ; \mathrm{CO}-$

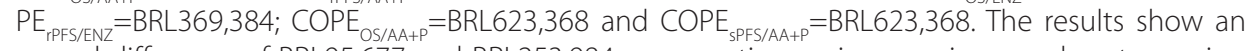
annual difference of BRL95,677 and BRL253,984, representing an increase in annual costs ranging from $18.13 \%$ to $68.76 \%$, depending on the considered outcome. Conclusion: The results indicated that ENZ when compared to AA+P provides a lower COPE $\left(C O P E_{E N Z}\right.$ is lower than $C O P E_{A A+P}$ for both outcomes analyzed: OS and rPFS).
\end{abstract}

\section{Introdução}

De acordo com dados globais de 2012, o câncer de próstata foi considerado o segundo tipo de neoplasia mais comum e a quinta maior causa de morte por câncer entre homens (World Health Organization, 2012). No Brasil, sua incidência é estimada em 70,4 casos a cada 100.000 homens, com cerca de 68.800 casos novos diagnosticados em 2014 (Ministério da Saúde (Brasil), 2014). Representa o tipo mais comum de câncer entre os homens e, em termos de número de mortes, ocupa a segunda posição, atrás apenas do câncer de pulmão. Ao todo, 14.500 mortes devido ao câncer de próstata são registradas no Brasil todo ano, com taxas de 16 mortes a cada 100.000 habitantes (Costa, 2014).

Câncer de próstata resistente a castração metastático (CPRCM) é definido como a neoplasia que apresenta progressão apesar dos níveis de testosterona circulante baixos (Tan \& Haaland, 2014). Até o início da última década, o CPRCM era considerado refratário à hormonioterapia, e com proliferação celular independente do bloqueio do receptor androgênico (Rodrigues et al., 2014). Com o avanço no desenvolvimento de novas tecnologias foi possível o desenvolvimento de dois novos compostos para o tratamento de CPRCM, ainda com atuação na sinalização do receptor androgênico, a enzalutamida e o acetato de abiraterona (Mottet et al., 2014).

A enzalutamida, previamente conhecida como MDV3100, é um inibidor da cascata de sinalização do receptor de androgênio, indicado para o tratamento do CPRCM em pacientes adultos que tenham recebido tratamento com docetaxel. De acordo com dados da literatura, comparado ao placebo, enzalutamida prolongou a sobrevida global (SG) e sobrevi- da livre de progressão radiológica (SLPr), além de melhorar a qualidade de vida e retardar o desenvolvimento de complicações ósseas (Astellas Farma Brasil Importação e Distribuição de Medicamentos Ltda., 2015; Scher et al., 2012).

$O$ acetato de abiraterona é também indicado para o tratamento de CPRCM em pacientes que receberam quimioterapia prévia com docetaxel. É primariamente conhecido por inibir seletivamente a CYP17, enzima necessária para a biossíntese de androgênios (Janssen-Cilag Farmacêutica Ltda., 2015). De acordo com os resultados da literatura, o uso da abiraterona proporciona maior queda no antígeno prostático específico (PSA) e prolonga a SG e a SLP nesses pacientes quando comparado com prednisona mais placebo. (Bono et al., 2011) Contudo, estudo de comparação indireta do acetato de abiraterona versus enzalutamida sugeriu vantagens para o uso da enzalutamida na avaliação de desfechos secundários (progressão e taxa de resposta do PSA e SLPr para pacientes com CPRCM pós-docetaxel) (Tan \& Haaland, 2014).

Análises econômicas em saúde têm como princípio a comparação de duas estratégias de tratamento levando em consideração custos e desfechos relacionados à doença de interesse. Dois tipos de análises comumente aplicadas são as análises de custo-efetividade e custo-utilidade. Nestas análises a diferença de custos de duas estratégias de tratamento (custo incremental) é comparada com a diferença de desfechos clínicos (efetividade incremental) (Drummond et al., 1997). O cálculo do custo incremental leva em consideração o custo com as drogas e os custos evitados pelo uso das mesmas, por exemplo, hospitalização ou qualquer outro evento evitado pelo seu uso (Drummond et al., 1997). Já a efetividade incremental é mensurada pelo número de anos 
de vida salvos pelo uso de uma droga em comparação à outra e, no caso das análises de custo-utilidade, o número de anos de vida salvos com qualidade (Drummond et al., 1997). A mensuração de todos os parâmetros clínicos e econômicos necessários para a elaboração deste tipo de análise é complexa e demanda tempo. Alguns autores vêm defendendo o desenvolvimento de análises menos complexas e mais amigáveis para médicos e gestores, a chamada análise de custo por evento evitado, em inglês, Cost of Preventing an Event (COPE) (Maharaj, 2008).

O COPE é uma aproximação da análise de custo-efetividade e pode ser utilizado para avaliar um novo medicamento para o qual uma avaliação econômica de custo-efetividade não está disponível, mas existe um estudo controlado randomizado (ECR) (Maharaj, 2008).

Assim, o presente estudo tem como objetivo desenvolver uma análise do COPE com enzalutamida e acetato de abiraterona no tratamento do CPRCM após quimioterapia, sob a perspectiva do Sistema de Saúde Suplementar no Brasil.

\section{Métodos}

Para calcular o COPE (Equação 1: Cálculo do custo por evento evitado (COPE) (Maharaj, 2008)) e mensurar o impacto econômico da enzalutamida comparada ao acetato de abiraterona no tratamento de homens com CPRCM pós-docetaxel, dois parâmetros foram necessários: (1) número necessário a tratar (NNT) e (2) custo anual de tratamento (Maharaj, 2008).

O NNT representa o número de pacientes a serem tratados para que um paciente adicional evite um evento indesejado (Laupacis et al., 1988). Em nossa análise o evento indesejado foi a progressão da doença ou morte. Assim, o NNT foi calculado como o inverso da redução do risco absoluto de um evento (progressão da doença ou morte) obtida com o novo tratamento no horizonte de tempo de 1 ano (Equação 2) (Laupacis et al., 1988).

O custo anual de tratamento foi calculado para o horizonte de tempo de 1 ano, considerando somente custos de medicamentos (Equação 3).

O COPE tem como objetivo demonstrar ao pagador qual é o custo real para cada paciente que evita que o evento indesejado aconteça, ou seja, obtém sucesso com o tratamento. Por exemplo, se 100 pacientes são tratados ao custo total de $R \$ 100.000,00$ e, destes, 50 apresentam sucesso ao tratamento (permanecem vivos), o custo por evento evitado será de R\$2.000,00. Ou seja, apesar do custo de tratamento por paciente ser de $\mathrm{R} \$ 1.000,00$ o custo para se evitar que um paciente morra é de $\mathrm{R} \$ 2.000,00$, uma vez que é preciso que 2 pacientes sejam tratados para que 1 obtenha sucesso com o tratamento. Desta forma, quanto maior a taxa de sucesso ao tratamento, menor será o custo para o pagador por paciente com sucesso. No exemplo anterior, se a taxa de sucesso aumentasse de $50 \%$ para $80 \%$, o custo por evento evitado passaria a ser de $\mathrm{R} \$ 1.250,00$.

Esta análise demonstra para o pagador que um tratamento mais barato no curto prazo pode se tornar mais caro no longo prazo se tiver uma baixa taxa de sucesso e vice-versa (Maharaj, 2008).

A análise de COPE foi aplicada tanto para a enzalutamida quanto para a abiraterona, calculando para cada uma delas o custo associado com o tratamento medicamentoso para o CPRCM após quimioterapia e comparando o mesmo em relação à taxa de sucesso obtida pelo paciente ao longo do tratamento.

Os desfechos avaliados para avaliar o sucesso do paciente ao tratamento foram: SG e SLPr.

Equação 1: Cálculo do custo por evento evitado (COPE) (Maharaj, 2008)

COPE = Custo anual de tratamento *NNT

Equação 2: Cálculo do número necessário a tratar (NNT) (Laupacis et al., 1988)

NNT = 1/(Taxa de Sucesso Tratamento $_{\text {- Taxa de Sucesso }}$ controll )
Taxa de sucesso foi avaliada por dois desfechos:
sobrevida global em 1 ano e sobrevida livre
de progressão radiográfica em 1 ano.

Equação 3: Cálculo do custo anual de tratamento (CAT)

$C A T=$ Duração do tratamento (meses) * Doses por mês * Custo por dose

O intervalo de confiança 95\% (IC95\%) foi calculado para o NNT (Equação 4: Cálculo do IC95\% do NNT) (Altman \& Andersen, 1999). Consequentemente, foi possível calcular o IC95\% para o COPE.

Equação 4: Cálculo do IC95\% do NNT

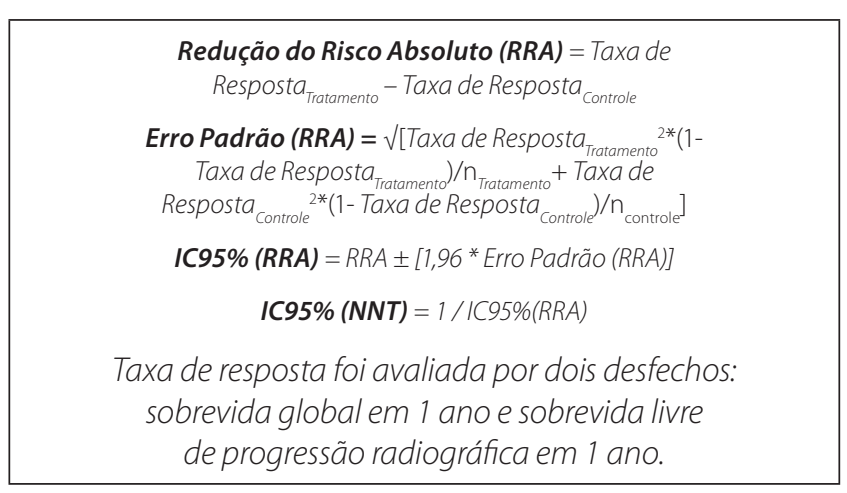

Onde: 
$\boldsymbol{n}=$ número de pacientes em risco no tempo da análise (12 meses)

Os desfechos de saúde considerados para avaliar a taxa de sucesso ao tratamento foram SG e SLPr, ambos avaliados 12 meses após o início do tratamento.

Na ausência de estudos que fizessem uma comparação direta entre enzalutamida e acetato de abiraterona, para alimentar a avaliação econômica com os dados de SG e SLPr foram selecionados os estudos pivotais de enzalutamida e acetato de abiraterona (Bono et al., 2011; Scher et al., 2012). Ambos os estudos selecionados incluíram pacientes com CPRCM após a quimioterapia.

Uma limitação deste tipo de avaliação é o fato de que os ensaios clínicos podem ter incluído populações diferentes de pacientes e ter adotado desenhos diferentes. Em termos de população de estudo, em Scher et al., 2012 (Scher et al., 2012) considerou-se como critérios de inclusão pacientes com diagnóstico histológico ou citológico de câncer de próstata, testosterona em níveis de castração (<50 ng/dL [1 ,7 nmol/L]), tratamento prévio com docetaxel e doença progressiva definida de acordo com o critério do Prostate Cancer Clinical Trials Working Group 2 (PCWG2), incluindo três valores crescentes para PSA ou progressão confirmada radiograficamente com ou sem aumento no nível de PSA. Já em Bono et al., 2011 (Bono et al., 2011), considerou-se principalmente o diagnóstico histológico ou citológico de câncer de próstata que foi previamente tratado com docetaxel, progressão da doença de acordo com o PCWG (os pacientes foram considerados com progressão da doença se apresentassem duas medições consecutivas indicando aumento na concentração de PSA acima do valor de referência) ou evidência radiográfica de progressão em tecidos moles ou osso com ou sem progressão nos valores basais de PSA, e privação androgênica em andamento, com níveis de testosterona sérica de 50 $\mathrm{ng} / \mathrm{Dl}$ ou menos ( $\leq 2 \mathrm{nmol} / \mathrm{L}$ ).

As características no baseline da população incluída em ambos os estudos foram avaliadas e concluiu-se que as populações são comparáveis, observando-se para o grupo enzalutamida um maior percentual de pacientes com extensão da doença para o pulmão (15,4\% enzalutamida versus 13\% acetato de abiraterona). A diferença principal entre os dois estudos está na escolha do grupo controle. Enquanto que no estudo do acetato de abiraterona todos os pacientes do grupo controle receberam prednisona mais placebo, no estudo com enzalutamida o uso de prednisona ou outro corticoide associado ao placebo era opcional.

Em resumo, os estudos individuais de cada produto parecem suficientemente semelhantes. As discretas diferenças entre os estudos não parecem claramente favorecer nenhum dos dois tratamentos. Assim, os dados individuais de cada estudo foram utilizados para alimentar as análises econômicas.

\section{Resultados}

\section{Dados clínicos}

Os principais resultados dos dois estudos selecionados foram obtidos para alimentar a análise econômica, como descrito a seguir (Bono et al., 2011; Scher et al., 2012).

\section{Enzalutamida: Scher e colaboradores, 2012 (Estudo AFFIRM) (Scher et al., 2012)}

800 pacientes foram randomizados para receber enzalutamida e 300 pacientes para receber placebo. O objetivo primário do estudo foi avaliar a SG dos pacientes.

A SG mediana foi de 18,4 meses no grupo tratado com enzalutamida versus 13,6 meses no grupo placebo (hazard ratio $(H R)=0,63 ; I C$ 95\%: 0,53 - 0,75; $p<0,001)$.

A SLPr, objetivo secundário do estudo, foi de 8,3 meses no grupo da enzalutamida 2,9 meses no grupo placebo (HR $=0,40 ; p<0,001)$

\section{Acetato de abiraterona: de Bono e colaboradores, 2011 (Bono et al., 2011)}

797 pacientes foram randomizados para receber acetato de abiraterona associada à prednisona e 398 para receber placebo mais prednisona. O objetivo primário do estudo foi avaliar a SG dos pacientes.

A SG mediana foi mais longa no grupo tratado com acetato de abiraterona associada à prednisona do que no grupo tratado com placebo associado à prednisona: 14,8 meses versus 10,9 meses ( $H R=0,65$; IC 95\%: 0,54-0,77; $p<0,001$ ).

A SLPr, objetivo secundário do estudo, foi de 5,6 meses no grupo do acetato de abiraterona versus 3,6 meses no grupo controle $(p<0,001)$.

Os resultados finais de sobrevida deste estudo foram publicados em setembro de 2012: com um acompanhamento mediano de 20,2 meses, a SG mediana foi mais longa no grupo tratado com acetato de abiraterona + prednisona: 15,8 meses versus 11,2 meses; $H R=0,74$ (IC 95\%: 0,64-0,86; $\mathrm{p}<0,0001$ ) (Fizazi et al., 2012).

Observa-se que os dados apresentados textualmente em ambos os estudos clínicos são os valores medianos obtidos ao longo do tempo de acompanhamento (Bono et al., 2011; Scher et al., 2012). Entretanto, para o cálculo do COPE no horizonte de tempo de 12 meses, precisamos dos dados de SG e SLPr neste mesmo horizonte de tempo. Estes dados puderam ser extraídos de curvas de Kaplan-Meier reportadas nos estudos clínicos, como apresentado na Tabela 1.

A Tabela 2 apresenta o NNT calculado para cada medicamento, utilizando a Equação 1: Cálculo do custo por evento evitado (COPE) (Maharaj, 2008) e a Equação 4: Cálculo do IC95\% do NNT.

A comparação do custo de ambos os medicamentos foi baseada nas doses disponíveis na bula de cada produto e 
Tabela 1. Percentuais de pacientes vivos e livres de progressão em 12 meses.

\begin{tabular}{lccl}
\hline & Enzalutamida & Placebo & Fonte \\
\hline $\begin{array}{l}\text { \% Pacientes vivos em 12 meses } \\
\text { radiográfica em 12 meses }\end{array}$ & $67 \%$ & $53 \%$ & AFFIRM (SCHER et al., 2012) \\
\hline & $30 \%$ & $10 \%$ & AFFIRM (SCHER et al., 2012) \\
\hline $\begin{array}{l}\text { \% Pacientes vivos em 12 meses } \\
\text { + predinisona }\end{array}$ & $60 \%$ & Placebo + predinisona & Fonte \\
\hline $\begin{array}{l}\text { radiográfica em 12 meses } \\
\text { racientes livres de progressão }\end{array}$ & $22 \%$ & $48 \%$ & de Bono, 2011 (BONO et al., 2011) \\
\hline
\end{tabular}

Tabela 2. Número necessário a tratar (NNT).

\begin{tabular}{lcccc}
\hline & Enzalutamida & IC95\% & $\begin{array}{c}\text { Acetato de abiraterona } \\
\text { + predinisona }\end{array}$ & IC95\% \\
\hline NNT: SG & 7,14 & $4,84-13,66$ & 8,33 & $5,01-24,79$ \\
\hline NNT: SLPr & 5,00 & $3,86-7,08$ & 8,33 & $5,59-16,39$ \\
\hline
\end{tabular}

NNT: número necessário a tratar; SG: sobrevida global; SLPr: sobrevida livre de progressão radiológica.

sustentadas por estudos publicados na literatura científica (Astellas Farma Brasil Importação e Distribuição de Medicamentos Ltda., 2015; Bono et al., 2011; Janssen-Cilag Farmacêutica Ltda., 2015; Scher et al., 2012).

\section{Dados econômicos Custo anual de tratamento}

A Tabela 3 apresenta as doses e frequências de administração para ambos os medicamentos.

O custo da enzalutamida considerado foi de $\mathrm{R} \$ 76,87$ por comprimido de $40 \mathrm{mg}$ e o do acetato de abiraterona de $\mathrm{R} \$$ 77,65 por comprimido com 250 mg. Para cada comprimido de $5 \mathrm{mg}$ de prednisona foi considerado o custo de $\mathrm{R} \$ 0,54$. Os valores utilizados para cálculo do custo foram baseados no preço fábrica com 18\% de ICMS reportados pela Câmara de Regulação do Mercado de Medicamentos (CMED), que representa a lista oficial de preços de medicamentos no Brasil (Agência Nacional de Vigilância Sanitária, 2015). Para ambos os tratamentos foi considerada a duração do tratamento de 8

Tabela 3. Dose e frequência de administração de acetato de abiraterona e enzalutamida.

\begin{tabular}{|c|c|}
\hline Enzalutamida & $\begin{array}{l}4 \text { comprimidos de } 40 \text { mg por } \\
\text { dia: } 120 \text { comprimidos por mês }\end{array}$ \\
\hline $\begin{array}{l}\text { Acetato de } \\
\text { abiraterona }\end{array}$ & $\begin{array}{l}4 \text { comprimidos de } 250 \mathrm{mg} \text { por } \\
\text { dia: } 120 \text { comprimidos por mês } \\
2 \text { comprimidos de prednisona } 5 \mathrm{mg} \\
\text { por dia: } 60 \text { comprimidos por mês }\end{array}$ \\
\hline
\end{tabular}

meses, obtida da mediana de duração de tratamento, observada nos estudos (Bono et al., 2011; Scher et al., 2012).

Os resultados estão apresentados na Tabela 4 e destacam uma economia de $\mathrm{R} \$ \mathbf{7 4 6 , 0 0}$ por paciente em tratamento com enzalutamida em comparação com acetato de abiraterona. Essa economia é ainda maior quando consideramos que o acetato de abiraterona exige a administração concomitante de prednisona, sendo a economia média anual igual a $\mathrm{R} \$ 1.006,00$ por paciente em tratamento com enzalutamida em comparação com acetato de abiraterona associado à prednisona.

\section{Custo por evento evitado (COPE)}

A Tabela 5 apresenta o COPE de cada medicamento. O horizonte de tempo da análise foi de 12 meses. Os resultados estão apresentados na Tabela 6 e mostram que o uso de acetato de abiraterona em combinação com prednisona proporciona um aumento no COPE em 12 meses, em relação ao uso de enzalutamida, que pode variar de 18,13\% a 68,76\%, dependendo do desfecho considerado.

Considerando o IC95\% calculado para o NNT baseado na SG, temos que a diferença entre o COPE de enzalutamida e acetato de abiraterona em combinação com prednisona pode variar de $\mathrm{R} \$ 17,4$ mil a $\mathrm{R} \$ 845,2$ mil ao longo de 1 ano. Isso representa um aumento nos gastos anuais que pode variar de 4,87\% a 83,76\%.

Considerando o IC95\% calculado para o NNT baseado na SLPr, temos que a diferença entre o COPE de enzalutamida e acetato de abiraterona em combinação com prednisona pode variar de $\mathrm{R} \$ 132,5$ mil a $\mathrm{R} \$$ 702,9 mil ao longo de 1 ano. Isso representa um aumento nos gastos anuais que pode variar de $46,40 \%$ a $134,34 \%$. 
Tabela 4. Custo unitário e anual de cada medicamento.

\begin{tabular}{lccccc}
\hline & Unidade & Custo Unitário & Unidades por mês & Custo Anual* & Diferença \\
\hline Enzalutamida & $\mathbf{4 0 ~} \mathbf{m g}$ & $\mathrm{R} \$ 76,87$ & $\mathbf{1 2 0}$ & $\mathrm{R} \$ 73.799$ & - \\
\hline $\begin{array}{l}\text { Abiraterona }+ \\
\text { prednisona }\end{array}$ & - & - & - & $R \$ 74.804$ & $\mathrm{R} \$ 1.006^{\mathrm{a}}$ \\
\hline $\begin{array}{l}\text { Abiraterona } \\
\text { Prednisona }\end{array}$ & $250 \mathrm{mg}$ & $\mathrm{R} \$ 77,65$ & 120 & $\mathrm{R} \$ 4.544$ & $\mathrm{R} \$ 260$ \\
\hline
\end{tabular}

a: enzalutamida versus acetato de abiraterona + prednisona; b: enzalutamida versus acetato de abiraterona.

* Duração do tratamento de 8 meses para ambos os tratamentos.

Tabela 5. Custo por evento evitado para enzalutamida e acetato de abiraterona + prednisona.

\begin{tabular}{|c|c|c|c|c|}
\hline & Enzalutamida & $\begin{array}{c}\text { Acetato de abiraterona } \\
\text { + predinisona }\end{array}$ & $\begin{array}{l}\text { Diferença } \\
\text { Anual }\end{array}$ & $\begin{array}{c}\text { Diferença } \\
\text { (\%) }\end{array}$ \\
\hline SG & $\mathrm{R} \$ 527.691$ & $R \$ 623.368$ & $R \$ 95.677$ & $18,13 \%$ \\
\hline SLPr & $R \$ 369.384$ & $R \$ 623.368$ & $\mathrm{R} \$ 253.984$ & $68,76 \%$ \\
\hline
\end{tabular}

SG: sobrevida global; SLPr: sobrevida livre de progressão radiológica.

\section{Análise de sensibilidade}

Os resultados apresentados anteriormente foram calculados com base nos parâmetros definidos para o caso base desta avaliação econômica. Entretanto, cenários secundários ao caso base foram analisados com o objetivo de avaliar o impacto no resultado com a alteração de parâmetros definidos chave da análise.

\section{Inclusão de custos com exames de acompanhamento em pacientes em uso de acetato de abiraterona em combinação com prednisona}

Além de comparar apenas o custo da enzalutamida com o acetato de abiraterona em combinação com prednisona, é necessário considerar os custos relacionados ao acompanhamento do paciente em uso de acetato de abiraterona. Segundo a própria bula do produto, os níveis séricos de transaminases e bilirrubina precisam ser determinados antes de iniciar o tratamento com acetato de abiraterona, a cada duas semanas durante os primeiros três meses de tratamento e, depois disso, mensalmente (Janssen-Cilag Farmacêutica Ltda., 2015). Esse acompanhamento não é obrigatório em pacientes em uso de enzalutamida.

Assim, para avaliar o impacto deste parâmetro nos resultados da avaliação econômica, incluímos o custo de 16 provas de função hepática (bilirrubinas, eletroforese de proteínas, TGO, TGP e Gama-PGT) por ano para os pacientes em uso de acetato de abiraterona em combinação com prednisona. O custo deste exame é de R\$ 77,58 segundo a Classi- ficação Brasileira Hierarquizada de Procedimentos Médicos (CBHPM) de 2015 (Associação Médica Brasileira, 2015).

Os resultados mostram uma economia de $R \$ 1.987,00$ por paciente em tratamento com enzalutamida em comparação com acetato de abiraterona. Essa economia é ainda maior quando consideramos que o paciente em uso de acetato de abiraterona também tem o custo com a prednisona, sendo a economia média anual de $\mathrm{R} \$ 2.247,00$ por paciente.

Além disso, os resultados apontam que o uso de acetato de abiraterona em combinação com prednisona proporciona um aumento nos custos por evento evitado em 12 meses que pode variar de 20,09\% a 71,56\%.

\section{Inclusão do custo da prednisona em pacientes em uso de enzalutamida}

É um fato que os pacientes em uso de acetato de abiraterona necessitam do uso concomitante da prednisona. Em pacientes em uso de enzalutamida, o uso concomitante de prednisona não é obrigatório. Entretanto, no estudo clínico que avaliou a eficácia e segurança de enzalutamida 30,1\% dos pacientes estavam usando algum corticoide no início do estudo (prednisona, dexametasona ou prednisolona) (Scher et al., 2012). No sentido de avaliar o impacto do uso de prednisona em pacientes em uso de enzalutamida, foi desenhado esse cenário no qual 30,1\% dos pacientes em uso de enzalutamida fazem uso concomitante de prednisona. 
Observa-se uma economia anual de $\mathrm{R} \$ 927,00$ por paciente em tratamento com enzalutamida em comparação com acetato de abiraterona.

\section{Discussão}

Em termos de avaliação econômica, o custo anual da enzalutamida no tratamento de homens com CPRCM após quimioterapia, quando comparado com acetato de abiraterona associado à prednisona mostra uma economia para o sistema privado de saúde brasileiro. Além disso, podemos dizer que o custo esperado para se evitar uma morte ou progressão em um ano (COPE) é menor com o uso da enzalutamida do que com o uso do acetato de abiraterona mais prednisona. Para que uma droga tenha um COPE menor do que outra, ela tem menor custo de tratamento ou melhor taxa de sucesso ou ambos. No caso da enzalutamida ambos os parâmetros foram observados. Assumindo que os gestores de saúde possuem recursos limitados, este resultado se torna notório, pois demonstra para o pagador que utilizando o mesmo montante ele será capaz de proporcionar melhores resultados clínicos para os pacientes, pois além de mais pacientes serem tratados por conta do menor custo de tratamento, mais pacientes terão sucesso com o tratamento devido à melhor taxa de sucesso.

A análise desenvolvida possui algumas limitações que devem ser levadas em consideração na interpretação dos resultados finais do estudo. A principal foi o uso de estudos individuais de cada produto (Bono et al., 2011; Scher et al., 2012), dada a inexistência de estudos de comparação direta entre as drogas. Para contornar essa limitação uma análise da população de cada estudo foi realizada e previamente apresentada, de forma a reduzir qualquer inconsistência nos resultados relacionada a comparação dos dados de eficácia dos estudos com populações divergentes. Uma limitação não resolvida, e que mantém alguma incerteza em relação aos resultados, foi a utilização de grupos controle diferentes - um com uso obrigatório de prednisona (Bono et al., 2011), e outro com uso opcional (atingindo aproximadamente 30\% dos pacientes) (Scher et al., 2012).

Outra limitação importante a ser ressaltada diz respeito aos eventos adversos. O COPE é uma avaliação econômica incompleta (Maharaj, 2008). Uma análise completa, como por exemplo a de custo-efetividade, consideraria não apenas o custo com as drogas, mas também todos os demais custos relacionados ao tratamento do paciente (por exemplo, o custo com os eventos adversos) (Maharaj, 2008). Entretanto, como os eventos adversos foram reportados de forma diferente entre os estudos, a inclusão dos custos destes eventos poderia gerar algum tipo de viés em favor de um dos tratamentos avaliados (Bono et al., 2011; Scher et al., 2012). No entanto, é importante ressaltar que esses eventos existem. Além disso, nos pacientes em tratamento com acetato de abiraterona, não podemos esquecer dos eventos relacionados ao uso da prednisona. O uso crônico de esteroides tem sido associado com o desenvolvimento de várias toxicidades, incluindo insuficiência adrenal, osteoporose, osteonecrose, hiperglicemia, hipocalemia, edema, dispepsia, aftas, hipertensão, catarata, glaucoma, insônia, miopatia e imunossupressão (Mantecorp Indústria Química e Farmacêutica Ltda., 2009).

Outra limitação não menos importante diz respeito à extrapolação dos dados dos estudos clínicos para o mundo real. O NNT foi calculado com base em dados de estudos clínicos controlados e não foi feita nenhuma análise para inferir se os resultados encontrados nos estudos clínicos se repetem no mundo real (Bono et al., 2011; Scher et al., 2012).

Apesar das limitações descritas, os tomadores de decisão podem usar o COPE como ferramenta para decisão sobre uso de novas drogas, realizando uma análise mais simples, rápida e amigável para todos os que as análises de custo-efetividade.

\section{Conclusão}

Os resultados apontaram para um menor custo por evento evitado em 12 meses quando utilizada a enzalutamida, em comparação ao acetato de abiraterona em associação com prednisona.

\section{Referências bibliográficas}

Agência Nacional de Vigilância Sanitária (Anvisa). Secretaria executiva. Lista de preços de medicamentos: preço fábrica e preço máximo de venda ao governo - CMED. 2015.

Altman DG, Andersen PK. Calculating the number needed to treat for trials where the outcome is time to an event. BMJ. 1999;319(7223):1492-5.

Astellas Farma Brasil Importação e Distribuição de Medicamentos Ltda. Xtandi" (enzalutamida) [Bula]. Responsável técnico: Sandra Winarski. São Paulo: 2015

Associação Médica Brasileira (AMB). Classificação Brasileira Hierarquizada de Procedimentos Médicos (CBHPM). São Paulo: AMB; 2015.

Bono J, Logothetis C, Molina A, Fizazi K, North S, Chu L, et al. Abiraterone and increased survival in metastatic prostate cancer. N Engl J Med. 2011; 364(21): 1995-2005.

Costa IC, Jerez-roig J, Souza DL, Medeiros PF, Barbosa IR, Curado M, et al. Future burden of prostate cancer mortality in Brazil: a population-based study. Cad Saude Publica. 2014; 30(11): 2451-2458.

Drummond MF, O'Brien B, Stoddart GL, Torrance GW. Methods for the Economic Evaluation of Health Care Programmes. 2nd ed. Oxford: Oxford University Press; 1997

Fizazi K, Scher HI, Molina A, Logothetis CJ, Chi KN, Jones RJ, et al. Abiraterone acetate for treatment of metastatic castration-resistant prostate cancer: final overall survival analysis of the COU-AA-301 randomised, double-blind, placebo-controlled phase 3 study. Lancet Oncol. 2012; 13(10): 983-92. 
Fonseca RP, Fernandes Junior AS, Lima VS, Lima SS, Castro AF, Horta HL, et al. Recidiva bioquímica em câncer de próstata: artigo de revisão. Rev. Bras. Cancerol. 2007; 53(2): 167-172.

Janssen-Cilag Farmacêutica Ltda. Zytiga ${ }^{\text {TM }}$ (acetato de abiraterona) [Bula]. Responsável técnico: Marcos R. Pereira. São Paulo: 2015.

Laupacis A, Sackett DL, Roberts RS. An assessment of clinically useful measures of the consequences of treatment. N Engl J Med 1988; 318(26): 1728-33.

Maharaj R. Adding cost to NNT: the COPE statistic. ACP J Club. 2008; 148(1):A8.

Mantecorp Indústria Química e Farmacêutica Ltda. Meticorten` (prednisona) [Bula]. Responsável técnico: Lucia Lago Hammes. Rio de Janeiro:2009.

Ministério da saúde (Brasil). Secretaria de atenção à saúde. Instituto Nacional de Câncer (INCA). Programa nacional de controle do câncer da próstata: documento de consenso. 2002a. 24 p.

Ministério da saúde (Brasil). Secretaria de atenção à saúde. Instituto Nacional de Câncer (INCA). Coordenação de prevenção e vigilância (CONPREV). Câncer da próstata: consenso. Rio de Janeiro: INCA, 2002b. 20 p.

Ministério da saúde (Brasil). Instituto Nacional de Câncer José Alencar Gomes da Silva (INCA). Estimativa 2012: incidência de câncer no Brasil. Rio de Janeiro: INCA, 2011.
Ministério da saúde (Brasil). Instituto Nacional de Câncer José Alencar Gomes da Silva (INCA). Estimativa 2014: incidência de câncer no Brasil. Rio de Janeiro: INCA, 2014.

Mottet N, Bastian P, Bellmunt J, Van der Bergh R, Van Casteren N, Cornford P, et al. Guidelines on prostate cancer. Eur Urol 2014; 65(1)124-37.

Ndibe C, Wang CG, Sonpavde G. Corticosteroids in the management of prostate cancer: a critical review. Curr Treat Options Oncol. 2015; 16(2):6.

Rodrigues S, Doresa M, Metrogosa V, Rodrigues M, Neto Gomes P, Cabrita M, et al. Carcinoma da próstata metastático resistente à castração - novas abordagens terapêuticas. Acta Urológica Portuguesa. 2014; 31(1-2): 36-40.

Scher H, Fizazi K, Saad F, Taplin M, Sternberg C, Miller K, et al. Increased survival with enzalutamide in prostate cancer after chemotherapy. N Engl J Med. 2012; 367(13): 1187-1197.

Tan P, Haaland B, Montero A, Kyriakopoulos C, Lopes G. Hormonal Therapeutics Enzalutamide and Abiraterone Acetate in the Treatment of Metastatic Castration-Resistant Prostate Cancer (mCRPC) Postdocetaxel. Clin Med Insights Oncol. 2014; 8:29-36.

World Health Organization (WHO). International Agency for Research on Cancer. GLOBOCAN 2012: Estimated cancer incidence, mortality and prevalence worldwide in 2012 [Internet]. Disponível em: http:// globocan.iarc.fr/Pages/fact_sheets_population.aspx. 